\title{
Examining Eye Movements in Visual Search through Clusters of Objects in a Circular Array
}

\author{
Carrick C. Williams, \\ Mississippi State University
}

Alexander Pollatsek, and

University of Massachusetts, Amherst

Erik D. Reichle

University of Southampton

\begin{abstract}
Participants were asked to search for a complete $O$ in an array consisting of eight clusters of four Landolt $C$ s (i.e., $O$ s with a gap) arranged in a ring. The size of the gap in the $C$ s varied from cluster to cluster but was held constant within a cluster. The manual response time data were consistent with a serial self-terminating search. More importantly, eye movement data supported a serial processing model as (a) clusters were fixated serially (either clockwise or counterclockwise) on most trials and (b) fixation times on a cluster reflected processing time on that cluster and were unaffected by the gap size of either the prior or succeeding cluster. Furthermore, the pattern of fixation times on a cluster was similar to the pattern of response times in a secondary task where a single cluster was presented at fixation. These data extend the findings of Williams and Pollatsek (2007) in which search was through a linear sequence of clusters, and indicate that a serial search pattern through clusters of these kinds of objects is not confined to reading-like linear arrays.
\end{abstract}

\section{Keywords}

Eye Movements; Visual Search; Clusters

The complexity of most real-world scenes prevents the extraction of all of the relevant information in a single fixation. Thus, people tend to move their eyes several times per second to extract information from various points in the stimuli. This tendency is also true of many visual search tasks; people typically move their eyes to focus on the stimuli that could be the target of their search (e.g., Findlay, 1997; Findlay, Brown, \& Gilchrist, 2001). Although the processes of search have been heavily researched over the last few decades, the contribution of eye movements to search has been less well studied. Eye movements in visual search have been shown to be influenced by both data-driven and conceptually-driven processes. Visual saliency, a data-driven factor, has been shown to have an effect on early choices of where to fixate in a visual search (e.g., Donk \& van Zoest, 2008; van Zoest \& Donk, 2005; van Zoest, Donk, \& Theeuwes, 2004). However, it is also clear that there are top-down influences on where the eyes go in a search. One general finding is that eye

Please address correspondence to: Carrick C. Williams, Department of Psychology, Mississippi State University, PO Box 6161, Mississippi State, MS 39762, cwilliams@psychology.msstate.edu, Telephone: (662) 325-1146.

${ }^{2}$ Although the diagonal clusters were slightly closer to the starting point, only 2 of the 10 participants showed a preference for selecting those clusters first.

Portions of this work were presented at the 2011 annual meeting of the Vision Science Society in Napels, FL. 
movements tend to be directed toward items in the display that share features with the target of the search (Findlay, 1997; Hooge \& Erklens, 1999; Williams, 1967; Zelinsky, 1996). The fact that eye movements can be influenced by both data-driven and conceptually-driven factors is reflected in that computational models of scene viewing and visual search typically require both types of factors to explain how humans perform these tasks (e.g., Itti \& Koch, 2000; Henderson, Malcolm, \& Schandl, 2009; Nuthmann \& Henderson, 2012; Nuthmann, Smith, Engbert, \& Henderson, 2010; Wolfe, 1994, 2007; Zelinsky, 2008).

However, current models of how humans search through visual scenes are limited in their explanatory adequacy at least in part because neither "visual search" nor "scene viewing" are particularly well-defined tasks. For example, in the standard visual-search paradigm, subjects are shown a target object and then an array of visually similar distractor objects and asked to respond via a manual button press either "yes" if the target object is present in the array and "no" if it is not. Most often, the targets are characters (e.g., the letter $T$ ) in an array of other visually similar characters (e.g., $L$ s) that are constructed so that the target is not discriminable from the other characters using a single feature (e.g., a red $T$ embedded within an array of red and green $T \mathrm{~s}$ and $L \mathrm{~s}$ ) to avoid situations where the stimuli are processed in parallel, allowing the target to simply "pop out" of the display (Treisman, 1982; Treisman \& Gelade, 1980). However, because the characters are randomly scattered at different locations in the display, it is difficult to use the subjects' eye movements (which vary markedly across trials) to make inferences about how they are searching through the arrays to locate targets. Furthermore, although such arrays are representative of some visual-search tasks outside the laboratory, they do not resemble all such tasks (e.g., looking for some part of an object that is comprised of several parts; e.g., see Biederman, 1987). And of course these inherent limitations of the standard visual-search paradigm are even more pronounced in scene viewing, where the objects are more complex and varied and the search is more likely to be guided by knowledge of the scene being inspected (e.g., looking for automobiles on the ground rather than in the sky) and idiosyncratic strategies.

Williams and Pollatsek (2007) attempted to address some of these concerns by creating a visual-search paradigm in which one could reasonably predict the order of fixations to determine how closely fixation locations and fixation durations mirror ongoing processing during search. They had participants search for a complete $O$ where the distractors were $O$ s with a single gap ranging from very small $\left(0.032^{\circ}\right.$ of visual angle $)$ to large $\left(0.128^{\circ}\right.$ of visual angle) in one of the cardinal directions (i.e., Landolt Cs as in Hooge \& Erklens, 1999; see also Figure 1). The letters were arranged into eight clusters in a single horizontal line, with each cluster being comprised of four characters. Crucial to the design, the gap size of the $C \mathrm{~s}$ varied between clusters but was the same within a cluster, so that the difficulty of determining whether a cluster contained the target varied from cluster to cluster. Participants started with a fixation cross on the left side of the screen and searched the clusters in a leftto-right order (although they were not instructed to do so).

Because the clusters were fixated in an orderly pattern, one could examine how the processing difficulty of (a) the currently fixated cluster, (b) the previously fixated cluster, and (c) the subsequently fixated cluster affected the decision about when to make a saccade from one cluster to the next. The central finding was that the mean gaze duration on a cluster (i.e., the sum of the fixation durations on a cluster between when it was first fixated and when the eyes first moved to another cluster) was a function of the gap size of the currently fixated cluster, but was unaffected by the gap size of either the previously fixated cluster or the cluster about to be fixated. A companion search task was conducted in which there was a single cluster of four characters presented at fixation and only manual response times were recorded. The effect of cluster difficulty (i.e., the differences between the gap size conditions) was virtually identical for response times in this single-cluster task when the 
cluster did not contain the target and gaze durations on clusters that did not contain the target in the actual eye-movement experiment. It thus appears that gaze duration on a cluster in this experiment was a reliable measure of the time to determine whether a target was absent in the cluster.

These findings generally mirror those in reading, in which gaze duration on a word is a reliable indicator of the time to identify the word (Rayner, 1998; see also Vanyukov, Warren, Wheeler, \& Reichle, 2012). However, the generalizability of the Williams and Pollatsek (2007) findings to other visual-search and scene-viewing tasks might be called into question because (a) the clusters were word-like (e.g., comprised of linear arrays of characters) and (b) both the search order and general pattern of eye movements were so similar to those in reading (i.e., linear, from left to right). As a result, the current study was an attempt to determine whether the Williams and Pollatsek results would generalize to a search task in which the array was comprised of multi-component stimuli arranged in an orderly manner, but not to such a degree as to make the task analogous to reading. The current study employed the same task (i.e., searching for an $O$ with Landolt- $C$ s as distractors), but with two major differences: (1) in each cluster, the four characters were arranged in a $2 \times 2$ matrix and (2) the eight clusters were arranged in a circle rather than in a straight, horizontal line (see Figure 1). Thus, the individual clusters did not look like words, and the search order was not at all determined either explicitly or implicitly (the initial fixation point was in the center of the array).

The current study therefore explored the feasibility of using reading as a "platform" for explaining and predicting eye movements in other non-reading tasks. Because eye movements in reading have been both extensively studied (e.g., for reviews, see Rayner, 1998, 2009) and modeled (e.g., see Reichle, Rayner, \& Pollatsek, 2003), it would clearly be advantageous to apply what has been learned about the cognitive processes controlling eye movements during reading to better understand the cognitive processes controlling eye movements in tasks like visual search. More concretely, if eye movements in a less readinglike visual-search task appear to be consistent with those observed during reading, then one might hope to use reading data and models to inform our understanding of visual search. However, if eye movements in a less reading-like search task are decidedly different than those observed during reading, then our ability to use reading data and models to inform our understanding of visual search would be limited only to tasks that emulate reading. To preview our results, we found that, even using our less reading-like visual-search displays, the eyes moved in a orderly fashion (e.g., Parker, 1978), producing patterns of data that were similar to those observed using our previous, more reading-like search display (Williams \& Pollatsek, 2007).

\section{Method}

\section{Participants}

Ten participants were recruited from the Mississippi State University Psychology participant program and completed the experiment for partial course credit. Three other participants were lost due to the difficulty of maintaining an acceptable calibration. All had normal or corrected to normal vision.

\section{Design and Materials}

The experiment had two portions: multi-cluster search and single-cluster search. The multicluster search materials were similar to those created for Williams and Pollatsek (2007). There were eight clusters, each of which contained four Landolt $C$ s, except that the cluster containing the target contained three Landolt $\mathrm{Cs}$ and an $O$. The four characters were 
arranged in a $2 \times 2$ matrix with each $C$ having a randomly selected orientation. The eight clusters were arranged in a ring (see Figure 1 ) with each cluster being $1.00^{\circ}$ in size. Because we were primarily interested in the cluster-to-cluster eye movements, each cluster was positioned in the display so that it was equidistant from the two adjacent clusters (i.e., the distance from the center of one cluster to the center of its adjacent clusters was $2.99^{\circ}$ ). To accomplish this, the clusters in the cardinal positions were initially located at the vertices of an imaginary diamond shape whose vertices were located $3.98^{\circ}$ from the center of the display. The clusters on the diagonals were also placed at the vertices of an imaginary square that was aligned with the midpoints of the lines connecting two adjacent cardinal direction clusters. The vertices of this imaginary square were enlarged so that the diagonal clusters were $2.99^{\circ}$ from the adjacent clusters. Because the clusters were arranged in this manner, the clusters that were on the diagonals were $3.74^{\circ}$ from the center of the display. Importantly, our analyses were not dependent on the initial cluster selected, but rather on the movement of the eyes from one cluster to the next, across a distance that was held constant between any two spatially adjacent clusters.

There were three within-subjects factors in the multi-cluster search portion of the experiment: target presence (present or absent), cluster gap size $(1,2,3 \text {, or } 4 \text { pixels })^{1}$, and cluster location. At the viewing distance in the experiment, 30 pixels subtended 1 degree of visual angle, so that the gap sizes were $0.033,0.066,0.100$, and 0.133 degrees for the four gap size conditions. Half of the trials were target-present, with one of the clusters containing the target $O$, and half were target-absent trials. Two clusters of each gap size appeared in all search displays and each cluster gap size appeared equally often in the eight possible locations. When present, the target $O$ appeared equally often in all cluster gap sizes. There were a total of 128 multi-cluster search trials for each participant.

In addition to the multi-cluster search trials, participants were also presented with singlecluster trials. These trials presented a single cluster identical in size and format to one of the clusters in the multi-cluster trials. The cluster always appeared in the center of the screen. The target $O$ was present in half of the trials and all cluster gap sizes appeared equally often. There were 64 single-cluster trials.

\section{Procedure}

Upon arrival, participants were given the informed consent form to sign. Following the informed consent procedures, participants were told to perform their best to search for a complete $O$ among $C$ s and to make their responses on the button box ("Yes" if present or "No" if absent). Participants were then positioned in a chin rest and a 9-point eye tracking calibration was performed. Calibration was checked throughout the experiment and the experimenter recalibrated if necessary. If the experimenter determined that the calibration was sufficient, he or she initiated the search trial when the participant was looking at a central fixation box. The participant searched the display and indicated his or her response on the button box. The search display terminated with the response.

The 64 single-cluster trials were presented following all the 128 multi-cluster searches. Although all of the events in the single-cluster trials took place at fixation, eye positions were also monitored and the participant maintained his/her position in the chin rest. The entire experiment required approximately 1 hour to complete.

\footnotetext{
${ }^{1}$ Because of an equipment change, a screen resolution of $800 \times 600$ was used in the current experiment compared to $1600 \times 1200$ in Williams and Pollatsek (2007). To compensate, the current experiment used 1-, 2-, 3-, and 4-pixel gaps rather than 2-, 4-, 6-, and 8pixel gaps. However, the visual angles of the gaps, and stimuli in general, were approximately the same as those in Williams and Pollatsek.
} 


\section{Apparatus and analysis}

Eye tracking was performed with an ISCAN ETL-400 eye tracker operating at $120 \mathrm{~Hz}$. The system is accurate to less than $1^{\circ}$ of visual angle. Eye tracking data were entered into a computer running E-prime 1.2 software (Schneider, Eschman, \& Zuccolotto, 2002), which interpreted the data in coordinates consistent with the display.

In the succeeding analyses, eye position samples were averaged with the immediately preceding two samples and then consecutive individual samples were combined into fixations if they were within 4 pixels. Fixations that were less than $90 \mathrm{~ms}$ or greater than $4,000 \mathrm{~ms}$ were eliminated from the analysis. Blinks were also eliminated from the analysis.

\section{Results}

\section{Analysis of the Multi-Cluster Task}

Analysis of manual response times and accuracy-The first, most global, analyses of the data in this task are of the overall manual response times and errors (see Table 1). The response time data indicate a huge difference between target-absent and target-present trials, $t(9)=8.71, p<0.001$, Cohen's $d=3.45$. The average manual response time on targetpresent trials was only a little over half that of the response time on target-absent trials; this is consistent with a serial self-terminating search across clusters because the participants would find the target and stop the search after searching half of the clusters, on average. However, the differences in manual response times among the target-present conditions were not significant $(F<1)$ and there was no consistent pattern across the conditions. One likely reason for this finding is that the target cluster's composition was only one of the eight clusters in the display, and thus the response time data described in Table 1 would include the processing of other absent clusters as well as the target cluster, potentially masking any difference. The accuracy data indicated that there were more errors on targetpresent trials than on target-absent trials, $t(9)=2.40, p<0.05$, Cohen's $d=0.96$. Moreover, the error differences among the target-present trials was significant, $F(3,27)=3.42, M S_{e}=$ $0.006, p<0.05, \eta_{\mathrm{p}}^{2}=0.275$, with accuracy paradoxically decreasing as gap size increased, although accuracy remained high at all gap sizes. The finding of decreasing accuracy with increasing gap size is puzzling and failed to replicate the pattern from the linear display of Williams and Pollatsek (2007) where a non-significant trend went in the other direction.

The difference in accuracy between target-present versus target-absent trials indicates that participants missed some targets more than false alarming on absent trials However, the differences in accuracy among the target-present conditions are difficult to interpret, as one would predict that targets would stand out more in the presence of $C \mathrm{~s}$ with wider gap sizes. This raises the possibility that the judgment that a target is present in a cluster may be a complex mixture of actually detecting the target when it is in the fovea and estimating that it is in a cluster when it is in the parafovea by computing something like the sum of the gap sizes in a cluster (which would be greater the larger the gap sizes of the $C$ s surrounding the target). As a result, in the fixation analyses below, we will focus exclusively on the targetabsent trials.

Scan-path analyses-Each fixation was first assigned to the nearest cluster using Euclidean distance. Although the participants were not instructed about how to search for targets, visual inspection of their scan-paths indicated that they were reasonably consistent in how they inspected the clusters: Participants scanned the clusters either clockwise or counter-clockwise on a majority of trials. To quantify this, we calculated the proportion of inter-cluster saccades that moved the eyes in a systematic fashion in either a clockwise or counter-clockwise direction. The mean proportion of systematic saccades across participants 
was $0.95(S D=0.07$; range $=0.79$ to 1$)$. The participants also fixated the overwhelming majority of clusters, on average skipping only 0.03 of the clusters $(S D=0.04$; range $=0$ to 0.1 ). Example scan paths can be found in Figure 2.

Eye-movement data pre-processing-The analyses reported below therefore include only those trials in which the proportion of systematic saccades was at least 0.75 ; this selection criterion excluded 16 of the 640 trials, leaving $98 \%$ of the trials and increasing the mean proportion of systematic saccades to 0.96 . All fixations on the first and last clusters fixated during each trial were excluded because the saccade to the first cluster originated from the center of the display, and because fixations on the last fixated cluster coincided with the response that terminated the trial. Data from one cluster was also excluded because it was the recipient of a fixation more than 3,000 ms in duration. (Analyses not reported in this article but using all of the data showed very similar patterns of results.) This trimming left eye-movement data from 3,745 fixated clusters from target-absent trials.

Gaze duration-In Williams and Pollatsek (2007) and in most reading studies (see Rayner, 1998, 2009), gaze duration is taken to be a reliable index of the time taken to process a stimulus or word. In the present study, gaze duration is the sum of the duration of all fixations on a cluster between when it is first fixated and when a saccade leaves the cluster for the first time. As can be seen in Table 2, gaze duration on a cluster sensitively reflected the gap size of the $C \mathrm{~s}$ on the cluster for clusters that did not contain the target, $F(3$, $27)=39.87, M S_{e}=5,068, p<0.001, \eta^{2}=0.184$. (All reported ANOVAs used GreenhouseGeisser corrections for sphericity violations as necessary, but with non-corrected degrees of freedom being reported to avoid confusion.) Moreover, a Tukey's HSD test indicated that most of the six pair-wise comparisons of gaze durations on clusters having different gap sizes were also significant (all $p s<0.01$ ), with the only two exceptions being the differences between clusters having 2- versus 3-pixel and 3-versus 4-pixel gap sizes. Finally, the negatively accelerated function relating gap size to gaze duration is almost perfectly predicted by a logarithmic function: the correlation between the log of the gap size and the mean gaze duration was $r=-0.98$.

Other fixation duration measures-Three other fixation duration measures are reported in Table 2. First-fixation duration is the duration of the first fixation on a cluster and single-fixation duration is the duration of the fixation duration on a cluster when there was only one fixation on a cluster. Finally, total time is the sum of all fixation durations on a cluster, so that it is equal to the gaze duration plus the duration of any further fixations due to regressions back to the cluster after it has been exited. We should note that all of these measures only count those trials on which a fixation of the appropriate type was made for a particular cluster and are thus conditional on the cluster being fixated on the first pass through the array (i.e., not being skipped). However, because only 158 clusters were skipped across all trials, our analyses will not include the probability of skipping a target-absent cluster.

The results for all these measures were quite similar to those of gaze duration. As Table 2 shows, the mean values of all the other three fixation-duration measures decreased monotonically with increasing gap size [first-fixation duration: $F(3,27)=39.87, M S_{\mathrm{e}}=$ 2,084, $p<0.001, \eta^{2}=0.126$; single-fixation duration: $F(3,27)=58.04, M S_{\mathrm{e}}=4,507, p<$ $0.001, \eta^{2}=0.153$; total time: $\left.F(3,27)=49.39, M S_{\mathrm{e}}=14,675, p<0.001, \eta^{2}=0.246\right]$. As was true of gaze durations, pair-wise comparisons using Tukey's HSD tests indicated that most clusters of each gap size differed from each other on all three dependent measures (all $p s<0.05$ ), with the only two exceptions being the pair-wise comparisons involving clusters having 2-versus 3-pixel and 3- versus 4-pixel gap sizes. Also note in Table 2 that the mean 
durations of single fixations on a cluster were substantially larger than the mean durations of all first fixations. This suggests that at least some fixations that precede a refixation on the same cluster are driven by a quick signal to fixate the cluster from another viewing position, and that the signal is initiated more quickly than the signal to move the eyes to a new cluster. (We will revisit this finding in the General Discussion, but it is worth noting that a similar pattern is observed in reading, where the first of multiple fixations on a word is typically shorter than a single fixation on a word; e.g., see Rayner, Sereno, \& Raney, 1996.) The correlations between log gap size and mean first-fixation duration, single-fixation duration, and total time were $r=-0.97,-0.98$, and -0.98 , respectively (all $p$ s $<0.05$ ). Thus, not only is gaze duration on a cluster sensitive to the gap size, but so too is the duration of the initial fixation on a cluster. Finally, the total time measures are greater than the gaze duration measures ( $M=141,96,74$, and $61 \mathrm{~ms}$ for 1-, 2-, 3-, and 4-pixel gaps, respectively), indicating that participants made regressions back to previously fixated clusters. The fact that these mean differences were highly correlated with gap size $(r=-0.96 ; p<0.05)$ suggests that participants were doing some double-checking when they did return to a previously fixated cluster, with more effort being required to double-check clusters having smaller gaps.

Fixation probability and duration measures-Table 2 shows the total number of fixations per cluster (including regressions back to a cluster following the first pass) was also as a function of their gap sizes (1-, 2-, 3-, or 4-pixel gaps). Consistent with the total time data, clusters having smaller gaps were the recipients of more fixations than clusters having larger gaps, $F(3,27)=22.15, M S_{e}=0.034, p<0.001, \eta^{2}=0.249$. Pair-wise comparisons using Tukey's HSD test indicated that clusters having 1-pixel gaps were fixated more often that clusters having either 3- or 4-pixel gaps (both $p \mathrm{~s}<0.01$ ). As with the fixation-duration measures, there was a strong linear relationship between the mean number of fixations on clusters and their log gap sizes $(r=-0.99 ; p<0.01)$, consistent with the finding that gap size modulated both fixation durations and the propensity to refixate.

Table 2 also shows, as a function of cluster gap size, the probabilities of clusters being the recipients of exactly one fixation, and of fixations after inter-cluster regressions from other clusters. Clusters having smaller gaps were less likely to be the recipients of single fixations, $F(3,27)=17.85, M S e=0.004, p<0.001, \eta^{2}=0.228$. Again, comparisons using Tukey's HSD test indicated that, with the exception of gap size 3 versus 4, each cluster of a given gap size differed significantly from the other clusters with respect to the probability of being fixated exactly once (all $p s<0.05$ ). However, the effect of gap size on the number of regressions back to a cluster was less clear because the omnibus $F$-test was not significant, $F(3,27)=2.99, M S_{e}=0.005, p=0.094, \eta^{2}=0.033$.

Fixation locations-Table 3 shows the mean location of the first fixation on a cluster measured in terms of distances (in pixels) from the centers of the fixated clusters, as a function of cluster gap size. As can be seen in the table, it did not differ as a function of gap size of the $C \mathrm{~s}$ in the cluster $(F<1.88)$. The absence of any effect for how far the mean first fixation was from the center of the cluster suggests that information about cluster gap size was not available early enough (i.e., from the parafovea) to influence saccade targeting. (This conclusion is supported by the results reported in the next section.)

Table 3 also shows the mean locations of single fixations, of the initial fixations of clusters that were refixated, and of the first-pass refixations (i.e., excluding any refixations that occurred after inter-cluster regressions), all as a function of the gap size of the cluster being fixated. Although neither the locations of single fixations or refixations varied as a function of gap size (both $F \mathrm{~s}<1$ ), the initial fixation locations on clusters that were subsequently refixated were modulated by gap size, $F(3,27)=3.72, M S_{\mathrm{e}}=2.29, p<0.01, \eta^{2}=0.249$, 
with fixations on clusters with the smallest gap size being closer to center than the larger gap sizes. This pattern, in combination with the finding that clusters having smaller gaps sizes were more likely to be refixated, provides additional evidence of the inverse relationship between gap size and processing difficulty-that initial fixations on clusters with smaller gaps were more likely to result in refixations, even if those initial fixations were located closer to the centers of the clusters. Finally, although, on average, single fixations ( $M=$ 14.91) tended to be located closer to the centers of clusters than the first of two or more fixations $(M=16.22)$, this trend was not significant $(t<1.4)$. Similarly, on average, the refixations $(M=15.77)$ tended to be located closer to the centers of clusters than the initial fixations $(M=16.22)$, but again this trend was not significant $(t<1)$. These results suggest that, although the target of the second fixation on a cluster may have in at least some instances been the center of a cluster, there was probably a tendency to overshoot the target because the intended saccades were short (McConkie, Kerr, Reddix, \& Zola, 1988). However, it is also possible that, after an initial fixation far from the center, another possible target for the refixation may have been the opposite side of the cluster.

Spillover effects-We also examined whether the processing difficulty associated with clusters of a given gap size "spilled over" and inflated fixation durations on subsequent clusters. This analysis was performed as recommended by Lorch and Myers (1990): By completing a multiple regression on each subject's data using the gap sizes of the fixated clusters, the previously fixated clusters, and their possible interactions as predictor variables, and then completing within-group, two-tailed $t$-tests to determine if the mean regression coefficients were significant predictors of each of our dependent measures. Interaction terms were included in these analyses because of the possibility that, for example, spillover effects might be limited to instances where the fixated cluster is easy to process (i.e., has a large gap), allowing more of the attention "gradient" to be allocated between it and the previously fixated cluster. And to avoid possible artifacts due to skipping, we included only pairs of clusters that were fixated successively within trials.

The mean regression coefficients, the standard errors of these means, and the $t$-values associated with each of the coefficient means are shown in Table 4 . As the table shows, the gap size of the cluster being fixated affected the number of fixations on a cluster and all the fixation duration measures on that cluster (all $t \mathrm{~s}>4$, all $p \mathrm{~s}<0.01$, all $d \mathrm{~s}>1.39$ ). In contrast, there was little evidence of the gap size of the previously fixated cluster directly affecting these measures. As Table 4 shows, the gap size of the previously fixated cluster only modulated the probability of making a regression, $t(9)=2.74, p<0.05, d=0.87$, with none of the other coefficients differing significantly from zero (all $t \mathrm{~s} \leq 1.71$, all $p \mathrm{~s} \geq 0.12$ ). Similarly, although the interaction coefficient was also almost significant for regressions, $t(9)=2.17, p=0.058, d=0.69$, there was little evidence that the gap size of the previously fixated cluster interacted with the gap size of the fixated cluster to modulate any of the other dependent measures on the fixated cluster (all $t \mathrm{~s} \leq 1.61$, all $p \mathrm{~s} \geq 0.14$ ).

Parafoveal-on-foveal effects-Next, we examined the possibility that the difficulty associated with the processing of a parafoveal cluster might influence the time spent on the fixated cluster. There are claims that this kind of effect (often termed a parafoveal-on-foveal effect) is prevalent in reading (e.g., Kennedy \& Pynte, 2005; Kliegl, Nuthmann, \& Engbert, 2005); however, there is now considerable evidence that the only reliable such effects occur when the fixation on a word is within a character or two of the subsequent word and thus could be due to a mis-targeted saccade or inaccuracies in the eye-movement recording apparatus (Dreighe, 2011; Dreighe, Rayner, \& Pollatsek, 2008; Hyönä \& Bertram, 2004; for a review, see Schotter, Angele, \& Rayner, 2012). This analysis was done in the same manner as the one reported above on spillover effects. The mean regression coefficients, the 
standard errors of these means, and the $t$-value associated with each mean coefficient are shown in Table 5. As in the prior analysis, the gap size of the cluster being fixated affected the number of fixations on a cluster and all the fixation duration measures (all $t \mathrm{~s}>2.7$, all $p \mathrm{~s}$ $<0.05$, all $d \mathrm{~s}>0.88$ ), as well as the probability of the cluster being fixated exactly once, $t(9)$ $=2.96, p<0.05, d=0.94$. In contrast, there was little evidence of parafoveal-on-foveal effects: None of the other coefficients differed significantly from zero (all $t \mathrm{~s} \leq 1.52$, all $p \mathrm{~s} \geq$ 0.16 ). Our analyses thus provide no evidence that whatever difficulty might be associated with processing a parafoveal cluster interacts with the time spent processing and looking at the fixated cluster.

In summary, there is clear evidence that the gap size of the $C$ s in a cluster affects the ease of deciding that the target letter $O$ is not present in the cluster, and that differences in the relative ease of making this decision in turn affects both the likelihood of clusters being fixated one or more times, and the durations of any resulting fixations on those clusters. However, these effects appear to be quite localized, affecting the various measures on the cluster of interest but not affecting fixations on subsequently fixated clusters (i.e., no spillover effects) and not being affected by subsequently fixated clusters (i.e., no parafovealon-foveal effects).

\section{Analysis of the Single Cluster Task}

Manual response times-The analysis of the single-cluster task is much more straightforward, as the two primary dependent variables were manual response time and accuracy. As Table 6 indicates, manual response time increased with decreasing gap size in a manner similar to that for the fixation-duration measures on a cluster, $F(3,27)=13.39$, $M S_{e}=12,104, p<0.001, \eta_{p}{ }^{2}=0.598$. The linear trend was significant for both target-absent [slope $=-74.38 \mathrm{~ms} / \mathrm{pixel} ; t(9)=4.97, p<0.001$, Cohen's $d=1.58$ ] and target-present trials [slope $=-58.63 \mathrm{~ms} / \mathrm{pixel} ; t(9)=3.87, p<0.004$, Cohen's $d=1.22$ ]. The relationship between the log of gap size and mean response time for target-absent trials was fairly linear, $r=-0.93$. The small difference in slope between target-present and target-absent trials was not significant $(t=1.08, d=0.34)$ and was far from the $2: 1$ ratio one would expect if searching for a target within a given cluster was completed in a serial, and self-terminating manner. In addition, the $51 \mathrm{~ms}$ overall average difference in manual response times between the target-absent versus target-present trials was also far from significant, $F(1,9)=2.43, p>$ $0.10, \eta_{p}{ }^{2}=0.212$. This also indicates that the four characters in a cluster were usually not processed in series in the task, suggesting that they were instead processed in a more holistic, parallel manner, similar to how individual letters within short (4-6 letter) words are processed (McClelland \& Rumelhart, 1981; Reicher, 1969; Wheeler, 1970).

Accuracy-As can be seen in Table 6, accuracy in the single-cluster task was very high and did not differ across conditions (all $p$ s $>0.20$ ).

\section{Manual response times in target-absent single-cluster trials compared to} fixation-time measures in multi-cluster task-The manual response-time slope for target-absent trials in the single-cluster task $(-74.38 \mathrm{~ms} / \mathrm{pixel})$ was less than the gazeduration slope in the multi-cluster task $(-97.29 \mathrm{~ms} /$ pixel $)$; however, this difference was not significant, $t(9)=1.48, p>0.10$. A second possible comparison to the slope of the manual response times for the target-absent trials is the slope for the single-fixation durations $(-83.06 \mathrm{~ms} / \mathrm{pixel})$ because there was a (non-significant) trend for single fixations to be closer to the center of the clusters than initial fixations for all the trials. Again, these two slopes were not significantly different $(t<1)$. 
The above comparisons are somewhat problematic because: (a) participants began each trial in the single-cluster trials fixating closer to center of the cluster (single fixations in targetabsent trials were 11.7 pixels from the center versus 14.5 pixels for single-fixation trials in the multi-cluster search); (b) the single-cluster trials always followed the multi-cluster trials, and thus participants may have been more practiced in the single-cluster version of the search task; (c) there were many fewer observations in the single-cluster trials and thus those data appeared somewhat noisier. In spite of that, it appears that the gap-size effect for both single-fixation and gaze duration on a cluster are reasonably similar to that for the singlecluster target-absent data, and thus that both measures are quite predictive of the time to process a cluster.

\section{Discussion}

The overall pattern of data is quite clear. The overall response times in the multi-cluster task were consistent with the participants searching through the clusters serially and terminating their search when they successfully detected the target. The eye-movement data support this conclusion. First, on most trials, participants searched the array of clusters in a reasonably organized fashion (i.e., either clockwise or counterclockwise around the ring of clusters) similar to previous work with line drawing scenes (e.g., Parker, 1978) and in the naming of objects from pictures (e.g., Griffin, 2001; Meyer, Sleiderink, \& Levelt, 1998). Second, consistent with a serial search from cluster to cluster, all fixation time analyses indicated that fixation time on a cluster was a function of the gap size of the $C \mathrm{~s}$ in that cluster and, as our regression analyses indicated, was unaffected by the gap size of the $C$ s in either the cluster that was previously fixated or the cluster that was about to be fixated. Third, the effect of gap size on gaze durations on target-absent clusters in the multi-cluster task was quite similar to the response times to a target-absent cluster in the single-cluster task. Admittedly, this correspondence was not perfect, but that was likely because participants' fixation locations in the single-cluster task were closer to the center of a cluster and because of more variability in initial fixation locations in the multi-cluster task. Further, in both tasks, the time spent examining target-absent clusters were logarithmically related to the gap size of the $C \mathrm{~s}$ in the clusters.

Although our experiment was not designed to test whether the four characters within a cluster were processed serially or in parallel, there was no evidence of serial processing of characters within a cluster in our search task, and the pattern of data strongly suggested parallel processing of characters in our single-cluster task, much as individual letters appear to be processed in short words (e.g., McClelland \& Rumelhart, 1981; Reicher, 1969; Wheeler, 1970). Moreover, as mentioned earlier, the fact that the error rates in our search task were not a simple function of gap size (and even seemed to go the "wrong way" to a slight extent) also suggested that there was some parallel evaluation of the characters within a cluster. That being said, it is important to emphasize that the question of whether processing of characters within a cluster is serial or parallel is separate from that of whether processing of sequences of clusters is serial or parallel, and that the data from our task strongly suggest that the individual clusters were indeed processed one at a time, analogous to how individual words are thought to be processed during reading (see Reichle, Liversedge, Pollatsek, \& Rayner, 2009).

We should make clear, however, that although gaze duration on a cluster was a good index of the time required to process that cluster, we are not claiming either that processing of a cluster begins when the eyes land on the cluster or that it ends at the moment when the eyes leave the cluster. Instead, it is likely that the processing of a cluster begins before it is fixated and ends before a saccade moves the eye away from it. A precise explanation for why this is likely to be true can be provided using the E-Z Reader model of eye-movement 
control in reading as a framework for thinking about our task (Pollatsek, Reichle, \& Rayner, 2006; Reichle, Pollatsek, Fisher, \& Rayner, 1998; Reichle, Warren, \& McConnell, 2009).

The E-Z Reader model has two core assumptions. The first is that lexical processing is supported by a covert attention mechanism that processes one word at a time. (Because lowlevel information, such as the location of word boundaries, is encoded in parallel from across the visual field, the oculomotor system always has information about where to target saccades.) The second core assumption is that the "signal" to shift attention is decoupled from the "signal" to initiate saccadic programming. More specifically, the completion of an early stage of lexical processing (called $L_{1}$ ) on word $_{n}$ causes the oculomotor system to begin programming a saccade to word $_{n+1}$, whereas the completion of a subsequent stage of lexical processing (called $L_{2}$ ) on word ${ }_{n}$ causes attention to shift to word w $_{n+1}$. Although most of the details of the model are not important here (for an overview of the model, see Reichle, 2011), the $L_{1}$ versus $L_{2}$ distinction allows the model to explain parafoveal processing of

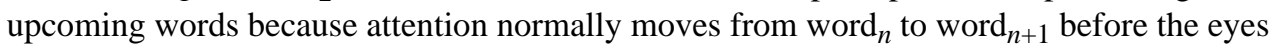
do. And for a similar reason, the model can also explain spillover effects: Because the $\mathrm{L}_{2}$ stage requires more time to complete from a difficult (e.g., low frequency) word, less time will be available for parafoveal processing of the next word, thereby inflating fixations on that word.

However, the fact that there were no spillover effects in our search task or that of Williams and Pollatsek (2007) suggests that the $\mathrm{L}_{1}$ versus $\mathrm{L}_{2}$ distinction may be limited to reading, and that a single "trigger" may provide the signal to move both attention and the eyes to the next cluster. However, because these two actions are not completed simultaneously (i.e., in the model, attention typically shifts in excess of $100 \mathrm{~ms}$ prior to the saccade onset; Schotter, Reichle, \& Rayner, 2013; see also Reingold, Reichle, Glaholt, \& Sheridan, 2012), the next cluster will normally receive some amount of parafoveal processing prior to being fixated. This in turn means that, although the processing of a cluster begins before it is fixated and ends before the eyes leave the cluster, these two times essentially cancel each other out, making the gaze duration on a cluster a virtually perfect index of the time taken to process the cluster. It is worth noting that Reichle, Pollatsek, and Rayner (2012) demonstrated the feasibility of this account using the variant of E-Z Reader model that was just described to simulate key aspects of both our task and the one used by Williams and Pollatsek (2007). The absence of spillover effects in our task would also be consistent with spillover effects reflecting, to some extent, integration of meaning across words (e.g., see Rayner, Warren, Juhasz, \& Liversedge, 2004), and that this type of integration is not necessary in our task.

It is also worth noting that the preceding account predicts that the gaze duration on a cluster will vary as the spatial relationship between the clusters changes. In the limit, if the clusters are far enough apart so that no useful information can be obtained from a cluster before it is fixated, then the gaze durations on the clusters should be longer than in the current experiments, where it is likely that some useful information was available before the clusters were fixated. This point is important because the absence of spillover and parafoveal-onfoveal effects in our data is not merely an artifact due to the clusters being far apart. Although we do not have acuity data to argue this point in the current experiment, both the initial characters of the upcoming cluster (i.e., cluster $N+1$ ) and the last characters of the previous cluster (i.e., cluster $N-1$ ) were clearly visible from the fixated cluster (i.e., cluster $N$ ) in Williams and Pollatsek's (2007) experiment. For that reason, gap-size information about clusters $N-1$ and $N+1$ could have influenced the fixation durations on cluster $N$. Therefore, the failure to find evidence of either spillover or parafoveal-on-foveal effects when clusters $N-1$ and $N+1$ were clearly visible strongly suggests that these null findingsand by extension our current findings-were not due to poor visual acuity, but rather the way in which spatial attention was allocated in the task. 
Our conclusions can be appreciated by way of contrast to the results of another experiment that used Landolt- $C$ stimuli but that were interpreted as providing evidence for parallel processing of stimuli during visual search (Trukenbrod \& Engbert, 2007; 2012). In that experiment, subjects viewed a matrix of densely spaced Landolt- $C$ stimuli and were instructed to move their eyes from a cued location along a trajectory indicated by the gap direction of each successive $C$. For example, upon fixating a stimulus having a gap facing upwards, the subject would move his/her eyes to the stimulus immediately above the one being fixated, determine the gap direction of that stimulus (e.g., facing left), and then continue moving his/her eyes accordingly (e.g., left) until a target (the letter $O$ ) was detected. The key finding of this experiment was that subjects seemed to process more than one stimulus at a time, so that, upon reaching a certain proximity to the target, their eyes often "jumped" over the last stimulus directly to the target. While we do not disagree with the interpretation of this result, we would also argue that the demands of this task are very different from those of ours because: (a) the stimuli were single $C$ s displayed close to each other and (b) the subjects quickly learned to use a single feature of those stimuli (the direction of their gaps) to move their eyes along a pre-defined path. This task is a relatively complex sequential selection task that may not generalize to visual searches like the one described in the current study. Because the searcher has to make multiple decisions about each distractor encountered (i.e., is it a target and if not, what direction does the stimulus require one to go next), the visual system may not operate the same in the Trukenbrod and Engbert task as in to other visual search tasks, especially ones that are made up of multicomponent items (e.g., letters in a word or clusters of symbols).

With that being said, let us conclude this article by returning full circle and situating our findings in the broader context of visual search and scene viewing. As indicated, our prior experiment (Williams \& Pollatsek, 2007) was motivated by our dissatisfaction with the tasks that have most often been used to study visual search and scene viewing, with standard search paradigms using random arrays of simple stimuli that make it extremely difficult to make sense of subjects' eye movements and that are unrepresentative of many real-world tasks, and with standard scene viewing paradigms being poorly defined and using complex stimuli that also make it difficult to make sense of subjects' eye movements. Although our prior experiment on some level addressed these limitations, it is also subject to criticism because of its obvious similarity to reading - a task that the average literate adult has spent years mastering and that might therefore be expected to have its own specific visual "routines" (see Reichle et al., 2012). Our current experiment represents an attempt to redress these limitations by using multi-component stimuli that are arranged in a novel configuration that is not too complex (e.g., random) to prevent its study through the use of eye movements. Thus, at a minimum, our experiment makes a methodological contribution by offering a task that is complex enough to be representative of the activities that people engage in when they search their environments for objects (or parts of objects), but simple enough that one can hope to understand such activities by taking advantage of the (relatively) systematic patterns of eye movements that are observed as subjects perform the task.

However, we believe that the main contribution of our current experiment is theoreticalthat the pattern of eye movements that were actually observed in our paradigm replicate those reported by Williams and Pollatsek (2007) and are therefore indicative of a serial selfterminating search in which gaze duration on a cluster or object is a reliable measure of the time needed to determine whether it contains a target or is a specific object. Thus, as a firstorder approximation, we contend that a majority of real-world search tasks are performed in a serial self-terminating manner. Although this is not to say that searches are never or cannot be done in some other manner (e.g., by allocating attention to multiple objects concurrently; Trukenbrod \& Engbert, 2012), the serial self-terminating search is probably the default in 
that it is both efficient and provides the searcher with a way of knowing which locations and/or objects have been examined and which have not (e.g., see Liu, Reichle, \& Gao, 2013). But of course, future work will be necessary to demonstrate this conclusively.

\section{Acknowledgments}

This research was supported by an NIH R01 Grant (HD26765) to the second author and Keith Rayner and an NIH R01 grant (HD053639) awarded to the third author.

\section{References}

Biederman I. Recognition-by-components: A theory of human image understanding. Psychological Review. 1987; 94:115-147. [PubMed: 3575582]

Drieghe, D. Parafoveal-on-foveal effects in eye movements during reading. In: Liversedge, SP.; Gilchrist, ID.; Everling, S., editors. Oxford Handbook on Eye Movements. Oxford, England: Oxford University Press; 2011. Manuscript in press.

Drieghe D, Rayner K, Pollatsek A. Mislocated fixations can account for parafoveal-on-foveal effects during reading. Quarterly Journal of Experimental Psychology. 2008; 61:1239-1249.

Donk M, van Zoest W. Effects of salience are short-lived. Psychological Science. 2008; 19(7):733739. [PubMed: 18727790]

Findlay JM. Saccade target selection during visual search. Vision Research. 1997; 37:617-631. [PubMed: 9156206]

Findlay JM, Brown V, Gilchrist ID. Saccade target selection in visual search: the effect of information from the previous fixation. Vision Research. 2001; 41:87-95. [PubMed: 11163618]

Griffin ZM. Gaze durations during speech reflect word selection and phonological encoding. Cognition. 2001; 82:B1-B14. [PubMed: 11672707]

Henderson JM, Malcolm GL, Schandl C. Searching in the dark: Cognitive relevance drives attention in real-world scenes. Psychonomic Bulletin \& Review. 2009; 16:850-856. [PubMed: 19815788]

Hooge ITC, Erkelens CJ. Peripheral vision and oculomotor control during visual search. Vision Research. 1999; 39:1567-1575. [PubMed: 10343822]

Hyönä J, Bertram R. Do frequency characteristics of nonfixated words influence the processing of fixated words during reading? European Journal of Cognitive Psychology. 2004; 16:104-127.

Itti L, Koch C. A saliency-based search mechanism for overt and covert shifts of visual attention. Vision Research. 2000; 40:1489-1506. [PubMed: 10788654]

Kennedy, A. Eye guidance in reading and scene perception. Oxford, England: Elsevier Science Ltd; 1998. The influence of parafoveal words on foveal inspection time: Evidence for a processing trade-off; p. 149-179.

Kennedy A, Pynte J. Parafoveal-on-foveal effects in normal reading. Vision Research. 2005; 45:153168. [PubMed: 15581917]

Kliegl R, Nuthmann A, Engbert R. Tracking the mind during reading: The influence of past, present, and future words on fixation durations. Journal of Experimental Psychology: General. 2006; 135:12-35. [PubMed: 16478314]

Liu Y, Reichle ED, Gao D-G. Using reinforcement learning to examine dynamic attention allocation during reading. Cognitive Science. 2013 Manuscript in press.

Liversedge S, Rayner K, White S, Vergilino-Perez D, Findlay J, Kentridge R. Eye movements when reading disappearing text: Is there a gap effect in reading? Vision Research. 2004; 44:1013-1024. [PubMed: 15031094]

McClelland JL, Rumelhart DE. An interactive activation model of context effects in letter perception: Part 1. An account of basic findings. Psychological Review. 88:375-407.

McConkie GW, Kerr PW, Reddix MD, Zola D. Eye movement control during reading: I. The location of initial eye fixations in words. Vision Research. 1988; 28:1107-1118. [PubMed: 3257013]

Meyer AS, Sleiderink AM, Levelt WJM. Viewing and naming objects: eye movements during noun phrase production. Cognition. 1998; 66:B25-B33. [PubMed: 9677766] 
Morrison RE. Manipulation of stimulus onset delay in reading: Evidence of parallel programming of saccades. Journal of Experimental Psychology: Human Perception and Performance. 1984; 10:667-682. [PubMed: 6238126]

Nuthmann A, Henderson JM. Using CRISP to model global characteristics of fixation durations in scene viewing and reading with a common mechanism. Visual Cognition. 2012; 20:457-494.

Nuthmann A, Smith TJ, Engbert R, Henderson JM. CRISP: A computational model of fixation durations in scene viewing. Psychological Review. 2010; 117:382-405. [PubMed: 20438231]

Parker RE. Picture processing during recognition. Journal of Experimental Psychology: Human Perception and Performance. 1978; 4:284-293. [PubMed: 660101]

Pollatsek A, Reichle ED, Rayner K. Tests of the E-Z Reader model: Exploring the interface between cognition and eye-movement control. Cognitive Psychology. 2006; 52:1-56. [PubMed: 16289074]

Rayner K. Eye movements in reading and information processing: 20 years of research. Psychological Bulletin. 1998; 124:372-422. [PubMed: 9849112]

Rayner K. Eye movements and attention in reading, scene perception, and visual search. The Quarterly Journal of Experimental Psychology. 2009; 62:1457-1506. [PubMed: 19449261]

Rayner K, Liversedge S, White S, Vergilino-Perez D. Reading disappearing text: Cognitive control of eye movements. Psychological Science. 2003; 14:385-388. [PubMed: 12807416]

Rayner K, Sereno S, Raney G. Eye movement control in reading: A comparison of two types of models. Journal of Experimental Psychology: Human Perception and Performance. 1996; 22:1188-1200. [PubMed: 8865619]

Rayner K, Warren T, Juhasz BJ, Liversedge SP. The effect of plausibility on eye movements in reading. Journal of Experimental Psychology: Learning, Memory, and Cognition. 2004; 30:12901301.

Reicher G. Perceptual recognition as a function of meaningfulness of stimulus material. Journal of Experimental Psychology. 1969; 81:275-280. [PubMed: 5811803]

Reichle, ED. Serial attention models of reading. In: Liversedge, SP.; Gilchrist, ID.; Everling, S., editors. Oxford handbook on eye movements. Oxford, UK: Oxford University Press; 2011. p. 767-786.

Reichle ED, Liversedge SP, Pollatsek A, Rayner K. Encoding multiple words simultaneously in reading is implausible. Trends in Cognitive Sciences. 2009; 13:115-119. [PubMed: 19223223]

Reichle ED, Pollatsek A, Fisher DL, Rayner K. Toward a model of eye movement control in reading. Psychological Review. 1998; 105:125-157. [PubMed: 9450374]

Reichle ED, Pollatsek A, Rayner K. Using E-Z Reader to simulate eye movements in non-reading tasks: A unified framework for understanding the eye-mind link. Psychological Review. 2012; 119:155-185. [PubMed: 22229492]

Reichle ED, Rayner K, Pollatsek A. The E-Z Reader model of eye-movement control in reading: Comparisons to other models. Behavioral and Brain Sciences. 2003; 26:445-526. [PubMed: 15067951]

Reichle ED, Warren T, McConnell K. Using E-Z Reader to model the effects of higher-level language processing on eye movements during reading. Psychonomic Bulletin \& Review. 2009; 16:1-21. [PubMed: 19145006]

Reingold E, Reichle ED, Glaholt MG, Sheridan H. Direct lexical control of eye movements in reading: Evidence from survival analysis of fixation durations. Cognitive Psychology. 2012; 65:177-206. [PubMed: 22542804]

Schneider, W.; Eschman, A.; Zuccolotto, A. E-Prime (Version 1.2) [Computer Software]. Pittsburgh, PA: Psychology Software Tools, Inc; 2002.

Schotter ER, Angele B, Rayner K. Parafoveal processing in reading. Attention, Perception, \& Psychophysics. 2012; 74:5-35.

Schotter ER, Reichle ED, Rayner K. Re-thinking parafoveal processing in reading: Serial-attention models can explain semantic preview benefit and $N+2$ preview effects. 2013 Manuscript submitted for review.

Treisman A. Perceptual grouping and attention in visual search for features and for objects. Journal of Experimental Psychology: Human Perception and Performance. 1982; 8:194-214. [PubMed: 6461717] 
Treisman A, Gelade G. A feature-integration theory of attention. Cognitive Psychology. 1980; 12:97136. [PubMed: 7351125]

Trukenbrod HA, Engbert R. Oculomotor control in a sequential search task. Vision Research. 2007; 47:2426-2443. [PubMed: 17662332]

Trukenbrod HA, Engbert R. Eye movements in a sequential scanning task: Evidence for distributed processing. Journal of Vision. 2012; 20:1-12.

Vanyukov PM, Warren T, Wheeler ME, Reichle ED. The emergence of frequency effects in eye movements. Cognition. 2012; 123:185-189. [PubMed: 22264379]

van Zoest W, Donk M. The effects of salience on saccadic target selection. Visual Cognition. 2005; 12(2):353-375.

van Zoest W, Donk M, Theeuwes J. The role of stimulus-driven and goal-driven control in saccadic visual selection. Journal of Experimental Psychology: Human Perception and Performance. 2004; 30(4):746-759. [PubMed: 15305440]

Wheeler D. Processes in word recognition. Cognitive Psychology. 1970; 1:59-85.

Williams CC, Pollatsek A. Searching for an O in an array of Cs: Eye movements track moment-tomoment processing in visual search. Perception \& Psychophysics. 2007; 69:372-381. [PubMed: 17672425]

Williams LG. The effects of target specification on objects fixated during visual search. Acta Psychologica. 1967; 27:355-360. [PubMed: 6062230]

Wolfe JM. Guided Search 2.0: A revised model of visual search. Psychonomic Bulletin \& Review. 1994; 1:202-238. [PubMed: 24203471]

Wolfe, JM. Guided Search 4.0: Current progress with a model of visual search. In: Gray, W., editor. Integrated models of cognitive systems. New York, NY: Oxford University Press; 2007. p. 99-119.

Zelinsky GJ. Using eye saccades to assess the selectivity of search movements. Vision Research. 1996; 36:2177-2187. [PubMed: 8776484]

Zelinsky GJ. A theory of eye movements during target acquisition. Psychological Review. 2008; 115:787-835. [PubMed: 18954205] 


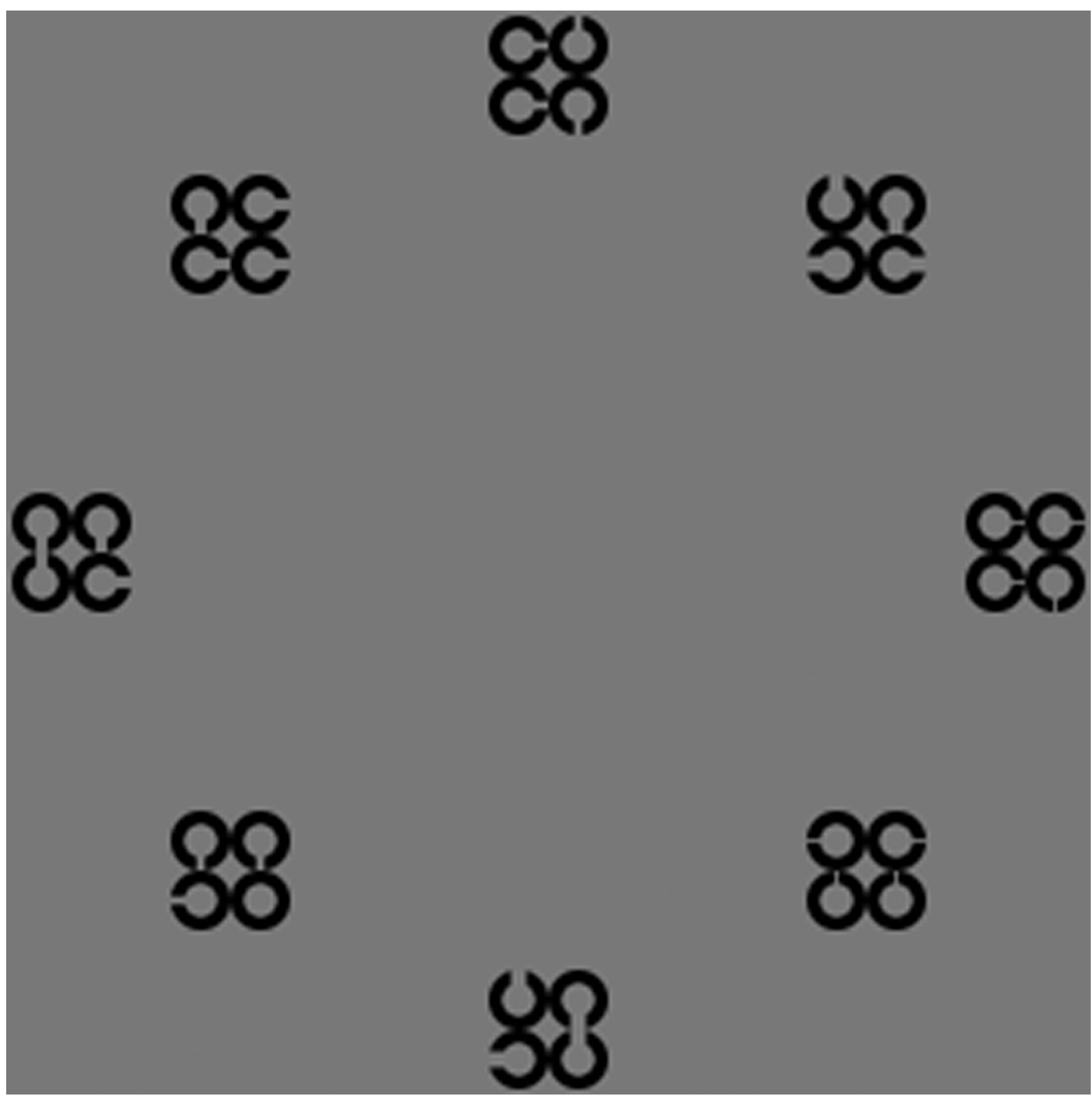

Figure 1.

Example stimuli used in a target-present trial. (The target $O$ is located in the cluster of Landolt $C$ s located in the bottom left of the display.) 


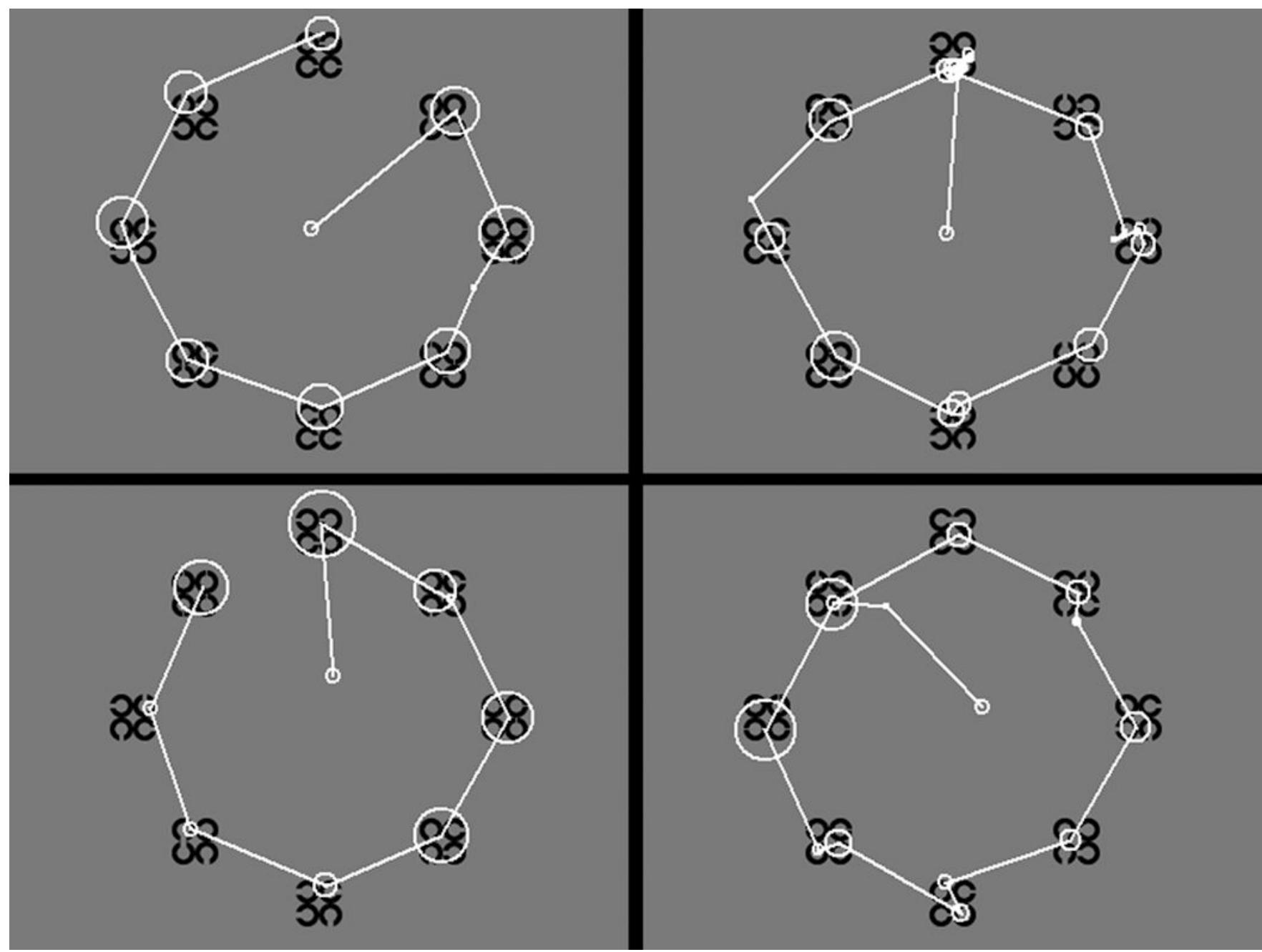

Figure 2.

Example scan-paths (indicated in white) from four target-absent trials. (Note that the sizes of the white circles denoting fixation locations represent the relative durations of those fixations.) 


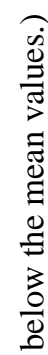

금

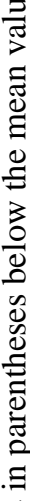

$\frac{0}{0}$

高

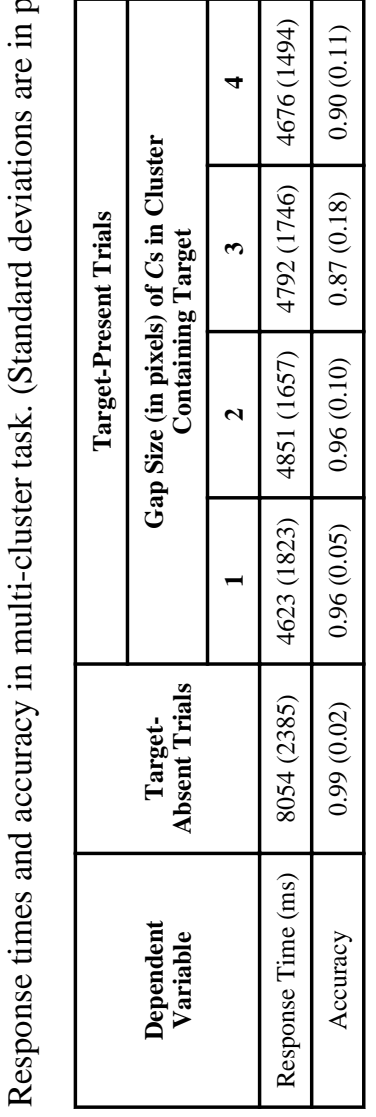

J Cogn Psychol (Hove). Author manuscript; available in PMC 2015 January 01. 


\section{Table 2}

Mean gaze duration, first- and single-fixation duration, total time, number of fixations, the probability of making exactly one fixation on a cluster, and the probability of a cluster being fixated after an inter-cluster regression as a function of the gap size of the $C$ s in a cluster. (Standard deviations are in parentheses.)

\begin{tabular}{|l|c|c|c|c|}
\hline \multirow{2}{*}{ Dependent Measure } & \multicolumn{4}{|c|}{ Cluster Gap Size (in pixels) } \\
\cline { 2 - 5 } & $\mathbf{1}$ & $\mathbf{2}$ & $\mathbf{3}$ & $\mathbf{4}$ \\
\hline Gaze Duration (ms) & $844(299)$ & $684(255)$ & $596(263)$ & $527(231)$ \\
\hline First-Fixation Duration (ms) & $661(250)$ & $544(210)$ & $495(223)$ & $449(189)$ \\
\hline Single-Fixation Duration (ms) & $778(287)$ & $633(242)$ & $571(262)$ & $501(217)$ \\
\hline Total Time (ms) & $984(313)$ & $780(252)$ & $669(277)$ & $588(252)$ \\
\hline Number of Fixations & $1.89(0.40)$ & $1.69(0.27)$ & $1.54(0.27)$ & $1.45(0.24)$ \\
\hline $\operatorname{Pr}($ Single Fixation) & $0.48(0.15)$ & $0.55(0.12)$ & $0.62(0.13)$ & $0.66(0.14)$ \\
\hline $\operatorname{Pr}($ Regression) & $0.19(0.18)$ & $0.16(0.13)$ & $0.14(0.12)$ & $0.12(0.11)$ \\
\hline
\end{tabular}




\section{Table 3}

Mean location (distance in pixels from cluster center) of first and second fixations. (Standard deviations are in parentheses.)

\begin{tabular}{|l|c|c|c|c|}
\hline \multirow{2}{*}{ Dependent Measure } & \multicolumn{4}{|c|}{ Cluster Gap Size (in pixels) } \\
\cline { 2 - 5 } & $\mathbf{1}$ & $\mathbf{2}$ & $\mathbf{3}$ & $\mathbf{4}$ \\
\hline All First-Fixation Locations & $14.42(2.97)$ & $14.96(3.16)$ & $15.37(3.22)$ & $14.88(3.36)$ \\
\hline Single-Fixation Locations & $14.21(4.33)$ & $14.36(4.14)$ & $14.77(4.23)$ & $14.70(4.46)$ \\
\hline First (of 2+) Fixation Locations & $15.26(1.61)$ & $16.40(2.66)$ & $17.17(2.84)$ & $16.07(2.92)$ \\
\hline Second-Fixation Locations & $16.21(3.41)$ & $15.50(4.74)$ & $16.03(3.55)$ & $15.33(4.36)$ \\
\hline
\end{tabular}




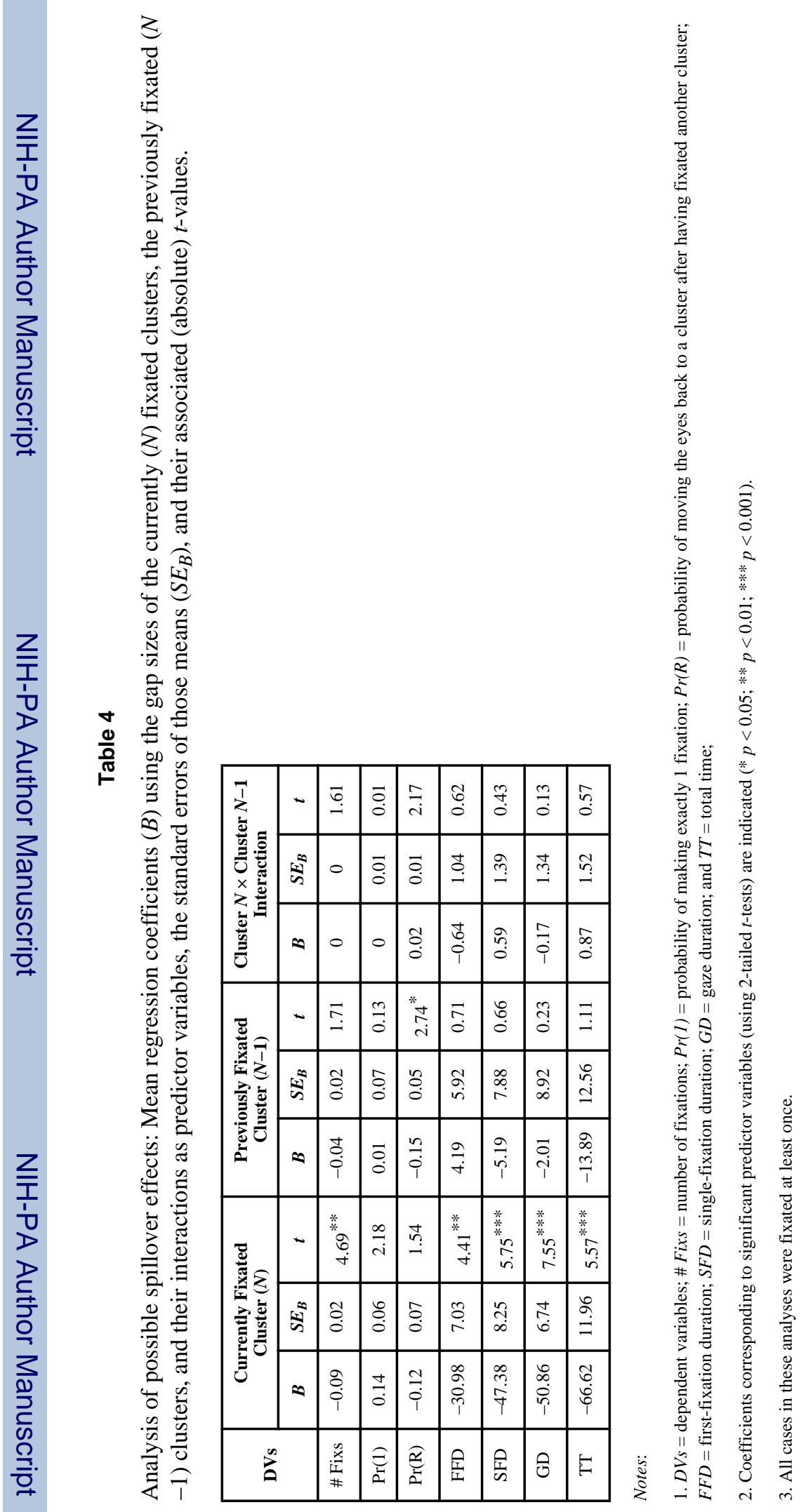




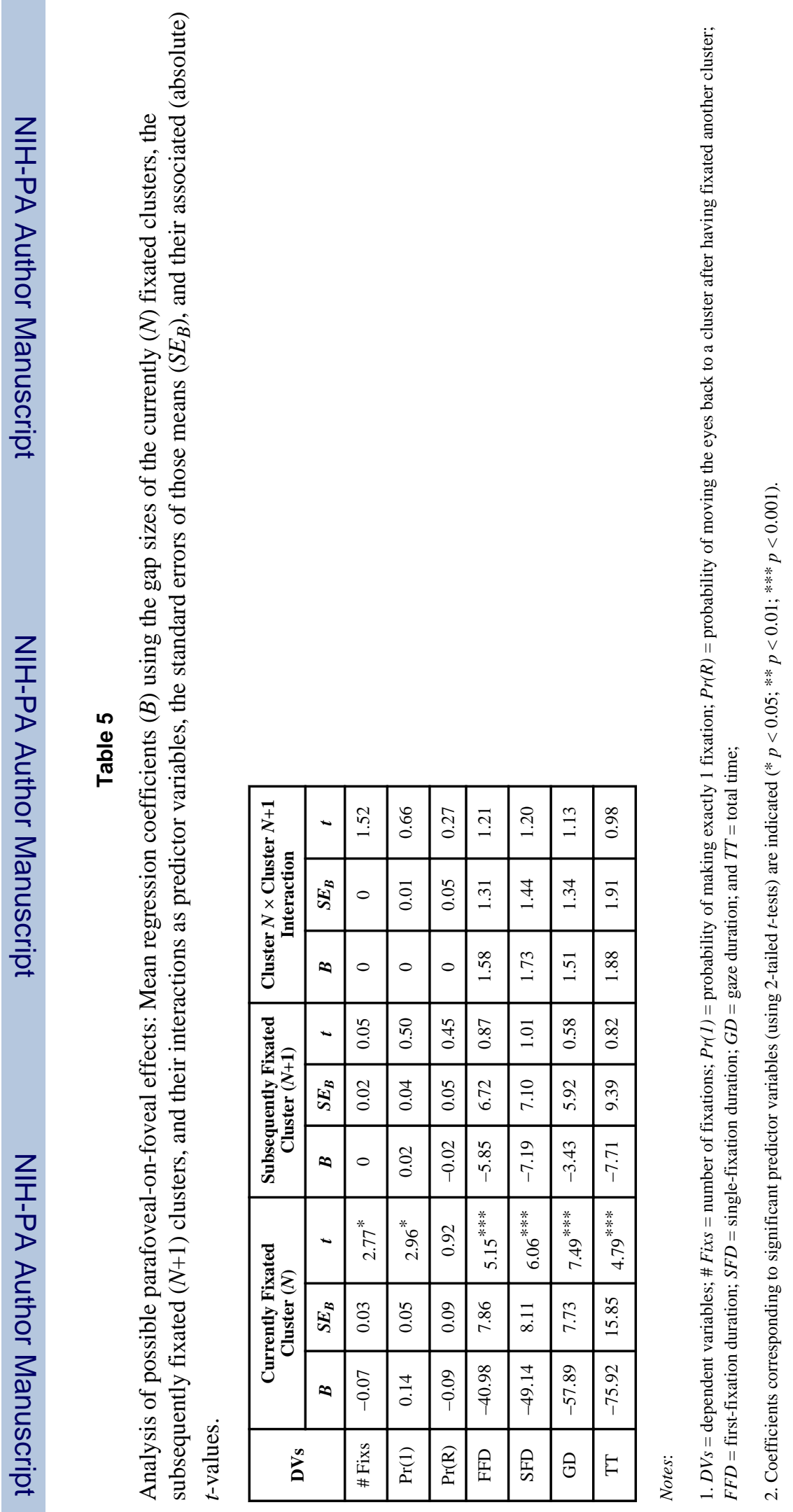

J Cogn Psychol (Hove). Author manuscript; available in PMC 2015 January 01. 


\section{Table 6}

Measures of processing for single-cluster search task. (Standard deviations are presented in the parentheses below the mean values.)

\begin{tabular}{|l|c|c|c|c|}
\hline \multirow{2}{*}{ Dependent Measure } & \multicolumn{4}{|c|}{ Cluster Gap Size (in pixels) } \\
\cline { 2 - 5 } & $\mathbf{1}$ & $\mathbf{2}$ & $\mathbf{3}$ & $\mathbf{4}$ \\
\hline Response time for Target-Absent Trials (ms) & $1346(468)$ & $1177(430)$ & $1200(535)$ & $1090(389)$ \\
\hline Response time for Target-Present Trials (ms) & $1253(487)$ & $1167(436)$ & $1117(429)$ & $1074(380)$ \\
\hline Accuracy for Target-Absent Trials & $0.99(0.02)$ & $0.99(.02)$ & $1.00(0)$ & $1.00(0)$ \\
\hline Accuracy for Target-Present Trials & $0.99(0.02)$ & $0.99(0.02)$ & $0.98(0.03)$ & $0.99(0.02)$ \\
\hline
\end{tabular}

